\title{
A Retrospective Analysis of R-MPV Plus Response-adapted Whole-brain Radiotherapy for Elderly Patients with Primary Central Nervous System Lymphoma
}

\author{
Yutaro Suzuki ${ }^{1}$, Naoto Imoto ${ }^{1}$, Shunichi Ishihara ${ }^{2}$, Shinji Fujiwara ${ }^{1}$, Rie Ito ${ }^{1}$, \\ Toshiyasu Sakai ${ }^{1}$, Satomi Yamamoto ${ }^{1}$, Isamu Sugiura ${ }^{1}$ and Shingo Kurahashi ${ }^{1}$
}

\begin{abstract}
:
Objective Few reports have described the real-world outcomes of rituximab, methotrexate (MTX), procarbazine, and vincristine (R-MPV) plus response-adapted whole-brain radiotherapy (WBRT) for elderly patients with primary central nervous system lymphoma (PCNSL). We evaluated the outcome of this regimen.

Methods We evaluated $>60$-year-old patients with newly diagnosed PCNSL who received R-MPV plus WBRT from January 2010 to December 2019 at Toyohashi Municipal Hospital. The patients' characteristics, regimen enforcement, response rate, survival, and toxicity were analyzed.

Patients Ten patients were consecutively enrolled. Their median age was 69 years old, and $60 \%$ had a performance status of 3 or 4 before induction therapy.

Results Seven patients achieved a complete response after induction, and all 10 patients achieved a complete response after consolidation. Seven received reduced-dose WBRT at $23.4 \mathrm{~Gy}$, and 2 received WBRT at 45 Gy. The median follow-up was 44.4 months; the 3-year progression-free survival and overall survival rates were $60 \%$ and $80 \%$, respectively; and the cumulative incidence of relapse was $40 \%$. The incidence of symptomatic delayed neurotoxicity was $70 \%$. Of the 7 patients who received reduced-dose WBRT, 4 (57\%) developed delayed neurotoxicity, including 1 severely affected patient. Only one patient survived without relapse and delayed neurotoxicity. The ratio of patients who developed relapse or delayed neurotoxicity that impaired daily life was $33 \%$ and $100 \%$ in the MTX high- and low-intensity groups, respectively.

Conclusion This regimen in elderly patients is unsatisfactory because of delayed neurotoxicity. We should consider maintaining an adequate MTX intensity, postponing or minimizing WBRT, and choosing high-dose consolidation therapy for select patients.
\end{abstract}

Key words: primary central nervous system lymphoma, R-MPV regimen, whole-brain radiotherapy, delayed neurotoxicity

(Intern Med Advance Publication)

(DOI: 10.2169/internalmedicine.7805-21)

\section{Introduction}

The optimal strategy for treating newly diagnosed primary central nervous system lymphoma (PCNSL) remains controversial. A typical treatment for newly diagnosed PCNSL is high-dose methotrexate (MTX)-based chemotherapy followed by whole-brain radiotherapy (WBRT) as consolida- tion $(1,2)$. However, adverse effects of WBRT, such as delayed neurotoxicity, are common, especially in elderly patients, and such adverse effects lead to a decreased quality of life $(2,3)$. One study showed that moderate to severely delayed neurotoxicity, defined as rapidly progressive subcortical dementia, occurred in $24 \%$ of patients (4). High-dose chemotherapy (HCT) supported by autologous stem cell transplantation (ASCT) as consolidation has been introduced

${ }^{1}$ Department of Hematology and Oncology, Toyohashi Municipal Hospital, Japan and ${ }^{2}$ Department of Radiology, Nagoya University Hospital, Japan

Received: April 21, 2021; Accepted: September 1, 2021; Advance Publication by J-STAGE: October 19, 2021

Correspondence to Dr. Naoto Imoto, imoto-naoto@toyohashi-mh.jp 
as an alternative to $\operatorname{WBRT}(5,6)$. In Japan, thiotepa was recently approved for treatment, further expanding the treatment options for PCNSL.

At our institution, we adopted the rituximab, MTX, procarbazine, and vincristine (R-MPV) regimen followed by response-adapted WBRT (ra-WBRT) and high-dose cytarabine (AraC) based on the results of a phase 2 study performed at the Memorial Sloan Kettering Cancer Center $(1,7)$. The study demonstrated a $79 \%$ complete response (CR) rate and median progression-free survival (PFS) and overall survival (OS) of 3.3 and 6.6 years, respectively, with minimally delayed neurotoxicity. However, few reports have described the efficacy and neurotoxicity of this regimen in real-world settings, especially in elderly patients.

We therefore retrospectively analyzed the outcomes of this regimen in elderly patients at our institution and reviewed the literature to determine whether or not our treatment strategy for PCNSL should be amended.

\section{Materials and Methods}

\section{Patients}

We retrospectively analyzed consecutive immunocompetent patients $>60$ years old with PCNSL who started the RMPV plus ra-WBRT regimen at Toyohashi Municipal Hospital from January 2010 to December 2019. We included all patients who received R-MPV plus WBRT, regardless of completion of or deviation from the regimen. Patients who received one course of another regimen before the R-MPV regimen were also included.

This study was approved by the institutional review board of Toyohashi Municipal Hospital.

\section{Institutional guideline for implementation of the regimen}

The R-MPV plus ra-WBRT regimen was performed based on the original study in which it was analyzed (7). In brief, the criteria for chemotherapy or WBRT dose reduction and pre-R-MPV treatment according to the patient's age, comorbidities, and general condition were not specified, being instead left to the discretion of each attending physician and through discussion at conferences. The rituximab, cyclophosphamide, doxorubicin, vincristine, and prednisolone regimen (R-CHOP) was chosen as the pre-R-MPV regimen according to a report describing its temporary efficacy against PCNSL $(8,9)$.

\section{Induction therapy}

We selected the R-MPV regimen as an induction therapy as follows: day 1 , rituximab at $375 \mathrm{mg} / \mathrm{m}^{2}$ intravenously; day 2, MTX at $3.5 \mathrm{mg} / \mathrm{m}^{2}$ intravenously (over 2 hours) and vincristine at $1.4 \mathrm{mg} / \mathrm{m}^{2}$ (capped at $2.0 \mathrm{mg}$ ); and days 2 to 8 , procarbazine at $100 \mathrm{mg} / \mathrm{m}^{2}$ per day, given during odd cycles. In the original study, rituximab was prescribed at 500 $\mathrm{mg} / \mathrm{m}^{2}$, but we reduced the dose to $375 \mathrm{mg} / \mathrm{m}^{2}$, which is commonly used in the treatment of malignant lymphoma. Standard hydration and leucovorin rescue were given in accordance with institutional guidelines. One cycle was 14 days. After five cycles of therapy, we performed magnetic resonance imaging (MRI) of the brain. Patients who achieved CR proceeded to consolidation therapy, while those who did not achieve $\mathrm{CR}$ received two additional cycles of the R-MPV regimen and consolidation therapy thereafter.

\section{Consolidation therapy}

Patients who achieved CR after five or seven cycles of induction received reduced-dose WBRT (rd-WBRT) at 23.4 Gy. Otherwise, WBRT at 45 Gy was offered. These doses were the same fractions used in the original trial. After WBRT, two courses of high-dose AraC were given at $3 \mathrm{~g} / \mathrm{m}$ /2/day on days 1 and $2(1,7)$.

\section{Follow-up assessments}

We acquired the patients' characteristics, course of treatment, adverse events during the regimen, response rate, PFS, OS, and delayed neurotoxicity from their medical records. The treatment response was assessed according to MRI with contrast enhancement. Because several patients did not undergo an eye examination or cerebrospinal fluid evaluation before treatment, the response based on the international criteria for PCNSL could not be determined in all patients (10). Instead, we used the RECIST version 1.1 evaluation as a generalized evaluation tool (11). CR was determined by the absence of the lesion, and partial response (PR) was determined by an at least $30 \%$ reduction in the sum or the maximum tumor diameters compared with baseline. Cases in which MRI showed shadows but the attending physician judged them to be scars from a brain biopsy were considered CR.

For the survival analysis, the time to event was calculated from the initiation of chemotherapy until death (OS), progression or death of any cause (PFS), or the date of the last follow-up in the intent-to-treat population. We also assessed the intensity of MTX as the relative dose intensity (MTXRDI; defined as the cumulative MTX dose divided by the estimated standard dose of MTX, with the standard dose set as $3.5 \mathrm{~g} / \mathrm{m}^{2}$ per cycle). Delayed neurotoxicity was defined as symptoms that appeared or deteriorated after completion of the series of this regimen without relapse. Adverse events and delayed neurotoxicity were assessed according to the Cancer Institute's Common Terminology Criteria for Adverse Events version 5.0. Delayed neurotoxicity was graded as follows: grade 1, MRI abnormality only; grade 2, symptoms that do not substantially interfere with life; and grade 3 , symptoms that substantially interfere with life.

Statistical analyses were performed using Kaplan-Meier survival plots with the EZR software program (Jichi Medical University Saitama Medical Center, Saitama, Japan) (12). 
(A)

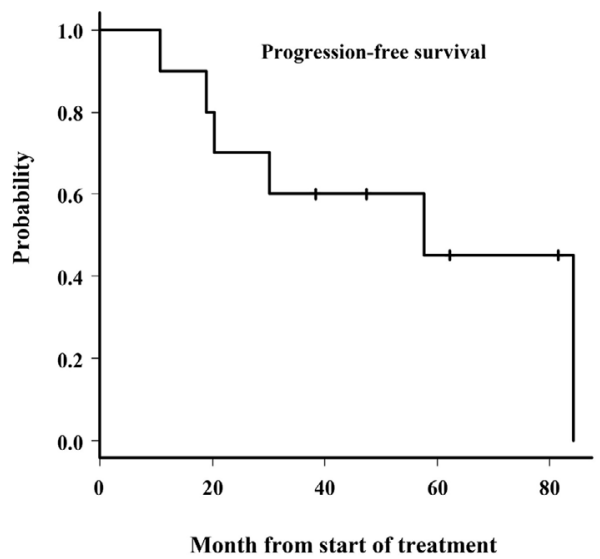

(B)

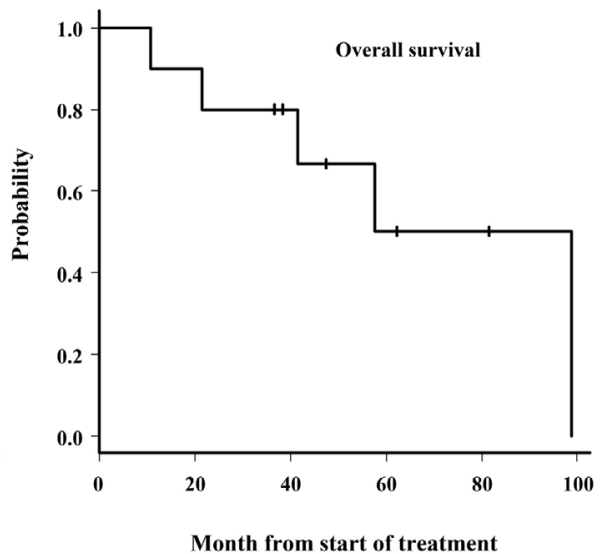

Figure. The progression-free survival and overall survival from the initiation of chemotherapy. (A) The progression-free survival. (B) The overall survival.

\section{Results}

\section{Patient characteristics}

We identified 10 patients with PCNSL who received the R-MPV plus WBRT regimen. All patients were $>60$ years old, and their median age was 69 (range, 62-77) years old. All but one patient was negative for human immunodeficiency virus (the status of Patient 6 was unknown because that patient was not tested). The performance status (PS) before treatment was 3 or 4 in 6 patients (Patients 2, 3, 5, 6, 9 , and 10) (60\%). Two patients received a pre-R-MPV regimen of R-CHOP to reduce the tumor burden (Patient 8 for an unknown reason and Patient 10 due to concerns regarding the safety of administering high-dose MTX over a long holiday). Patient 8 dropped out of the protocol after the second cycle of R-MPV for an unknown reason. Thus, nine patients completed the induction, and six of them improved to a PS of 0 to 2 after induction. Patient 5, who had lesions in the basal ganglion, thalamus, and cerebral peduncle, remained at a PS of 4 despite improvement of the lesions.

Seven patients received rd-WBRT at $23.4 \mathrm{~Gy}$, and 2 received WBRT at $45 \mathrm{~Gy}$. Five of 10 patients were excluded from 1 or all courses of high-dose AraC because of old age, a poor PS, or a continuous fever during the first course. Five patients completed treatment as scheduled in the protocol. The initial dose of high-dose MTX was reduced to 1 to $2 \mathrm{~g} /$ $\mathrm{m}^{2}$ for four patients (Patients 4, 7, 8, and 9). Three patients (Patients 2, 5, and 6) received a reduced MTX dose during treatment because of adverse events. One patient received high-dose $\mathrm{AraC}$ at $3 \mathrm{~g} / \mathrm{m}^{2}$ (Patient 3), and 5 patients received a reduced dose of 1 to $2 \mathrm{~g} / \mathrm{m}^{2}$.

\section{The response and survival}

Following the evaluation at the end of the five courses of the R-MPV regimen, six of nine patients achieved CR and proceeded to consolidation therapy. The remaining three pa- tients achieved PR and two additional courses of R-MPV. Of the three patients with PR, one achieved CR and the other two still showed PR following the additional courses. For the patients who were able to be evaluated, the CR rate for the completion of induction and consolidation was $70 \%$ and $100 \%$, respectively. The median follow-up period was 44.4 (range, 10.7-98.9) months, the 3-year PFS was $60.0 \%$ (median survival, 57.6 months), and the 3-year OS was $80.0 \%$ (median survival, 78.2 months) (Figure). Four patients $(40 \%)$ survived without relapse, and $3(30 \%)$ died of PCNSL. Patient 2 died of gallbladder cancer without relapse, and Patient 5 died of an unknown cause at home. At the time of this writing, Patient 9 was receiving palliative treatment after the second relapse. Patient 3 survived without relapse and delayed neurotoxicity. Table 1 shows the patients' characteristics and clinical outcomes.

We stratified the 10 patients into two groups: the highintensity group (MTX-RDI of $>0.80 ; n=6$ ) and the lowintensity group (MTX-RDI of $<0.50, n=4$ ). All patients in the high-intensity group reached CR after five or seven cycles of R-MPV and received rd-WBRT. In the low-intensity group, however, three of the four patients did not achieve CR after induction. Two of them received an additional 2 cycles (total of 7 cycles) of R-MPV and a higher dose of WBRT (45 Gy) (50\%) accordingly. The ratio of patients who relapsed or had delayed neurotoxicity that impaired their daily life was $33 \%$ in the high-intensity group but $100 \%$ in the low-intensity group.

\section{Adverse events during treatment}

Hematological adverse events of grade $>3$ during induction were neutropenia (Patients 2, 3, 4, 5, 7, 8, 9, and 10) (n $=8)$, anemia (Patients 2, 5, and 10) $(\mathrm{n}=3)$, and thrombocytopenia (Patient 10) $(\mathrm{n}=1)$. Adverse events during highdose AraC treatment were neutropenia (Patients 1, 2, 3, 4, 6, and 7) $(\mathrm{n}=6)$, anemia (Patients 4 and 6) $(\mathrm{n}=2)$, and thrombocytopenia (Patients 1, 3, 4, 6, and 7) ( $\mathrm{n}=5$ ). Nonhematological adverse events of grade $>3$ were sepsis $(\mathrm{Pa}-$ 


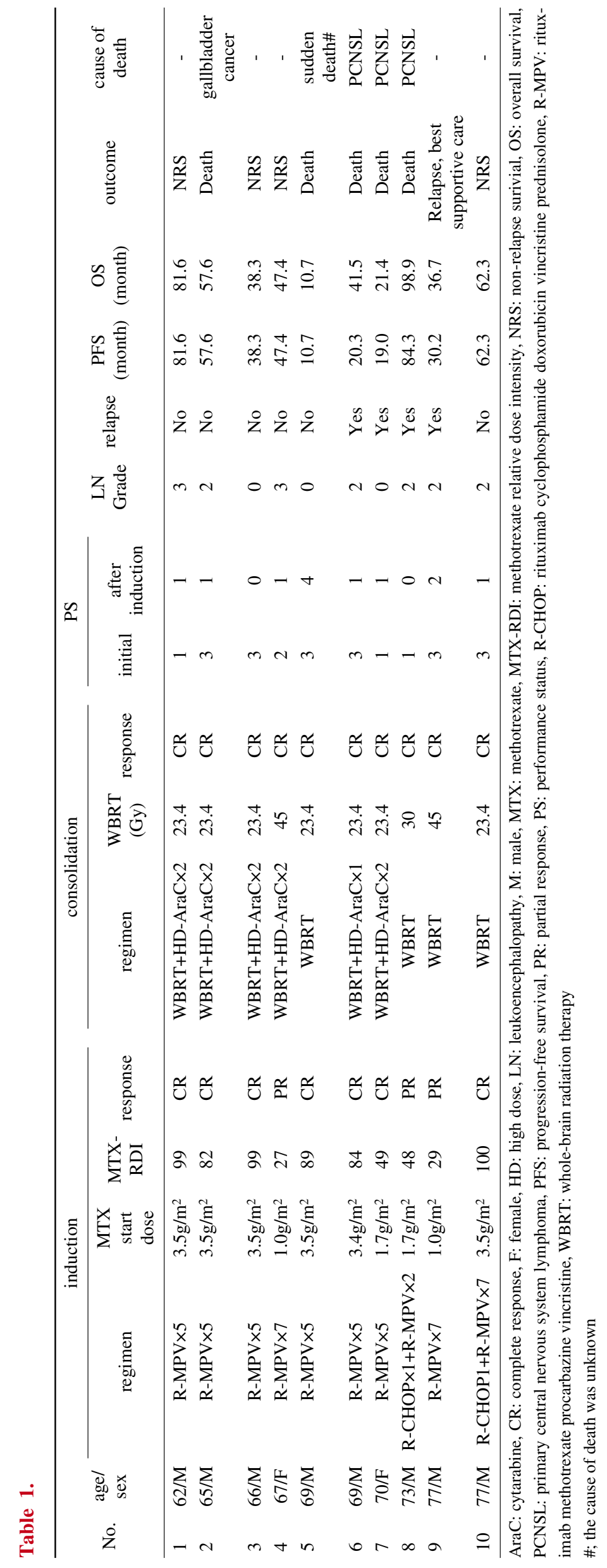


tients 9 and 10) $(\mathrm{n}=2)$, elevated alanine aminotransferase (Patients 2 and 6) $(\mathrm{n}=2)$, and hyponatremia (Patient 2) $(\mathrm{n}=$ 1) (all events occurred during induction). No life-threatening adverse events were observed during any course of the regimen.

\section{Delayed neurotoxicity}

Of the 10 patients who received WBRT, delayed neurotoxicity was observed in 7 . Of the 7 patients who received rd-WBRT at $23.4 \mathrm{~Gy}$, grade 2 and 3 delayed neurotoxicity was observed in 3 and 1 patient, respectively (57\%). Patient 1 had lesion-induced cognitive impairment before treatment. The Revised Hasegawa Dementia Scale (HDS-R) score was 11 before treatment but improved to 18 after the third course of R-MPV. After completion of the regimen, the doctor determined that the patient had no cognitive decline (an objective evaluation test was not done), and the patient returned to work. MRI showed leukoencephalopathy at six months after completion of consolidation, but the patient was asymptomatic. After two years, he developed irritability and deterioration of his cognitive function. These symptoms worsened, and he started rehabilitation. The HDS-R and Wechsler Adult Intelligence Scale scores were 12 and 59 (full-scale), respectively. After 3.5 years, he began to receive care services. After 4.5 years, he became prone to falls and showed signs of verbal abuse, violence, and disorientation, and his memory loss worsened, requiring treatment at a psychiatric hospital. His HDS-R score was 10 at that time. At the last follow-up six years after treatment, the patient was unable to walk, required a wheelchair at all times, and had considerable difficulty speaking. Patient 2 also had cognitive decline before treatment, and this patient's HDS-R score was 9 after the second course of R-MPV. The HDS-R score improved to 21 after consolidation. From three months after the end of treatment until his death of gallbladder cancer, the patient complained of forgetfulness and had MRI findings of leukoencephalopathy; however, he had no difficulties in daily life. Patient 6 complained of forgetfulness at one year and one month after treatment. Two months later, he relapsed. Although MRI was not performed at the onset of the forgetfulness, the forgetfulness was determined to be the result of delayed neurotoxicity because the symptoms at the time of relapse were pain and paralysis in the left lower back and left lower leg, and there was no worsening of the forgetfulness at that time. He was not evaluated using an objective cognitive test. Patient 10 had an HDS-R score of 20 before treatment and 27 after treatment. Two months after treatment completion, he complained of forgetfulness and walking instability. After six months, MRI showed leukoencephalopathy. His forgetfulness and walking instability worsened at four years and seven months, and he underwent a consultation with the Department of Neurology. His MiniMental State Examination (MMSE) score was 24. At the last follow-up, he complained of staggering, gait instability, difficulty differentiating between dreams and reality, being unable to walk much, bumping into objects, and being forget- ful; however, he was still able to live independently. All 3 patients who received WBRT at $\geq 30$ Gy had delayed neurotoxicity of grade $\geq 2$. Patient 4 had an HDS-R score of 21 before treatment. Her cognitive function began to deteriorate (with an HDS-R score of 11) at 9 months after the completion of treatment with MRI findings of leukoencephalopathy. She experienced gait instability that required walking assistance, urinary incontinence, incoherent conversation, hallucination, and soliloquy in addition to cognitive decline. After two years and three months, she developed a cerebral infarction and was transferred to another hospital. At the last follow-up of three years and three months after the treatment, she was bedridden, not speaking, and receiving gastrostomy feedings. Patient 8 complained of forgetfulness without impairment of daily life from one year and four months to the development of relapse at six years eight months. He was not evaluated using an objective cognitive test. Patient 9 had an HDS-R score of 26 and MMSE score of 23 before treatment, but these scores were not evaluated after treatment. He complained of diplopia and walking instability with MRI findings of leukoencephalopathy without relapse at one year and two months after completion of treatment. His symptoms continued until relapse.

\section{Discussion}

We investigated the real-world outcomes following treatment with the R-MPV plus ra-WBRT regimen for elderly patients with PCNSL. Our results indicated that this regimen had a good response rate: all 10 patients achieved CR at the end of the consolidation. However, four patients relapsed, and seven patients had delayed neurotoxicity after completing the regimen. Only one patient survived without relapse or delayed neurotoxicity. Despite the high response rates, the long-term outcomes were not satisfactory.

The R-MPV plus ra-WBRT regimen seems to be widely used in Japan, likely because this regimen is one of the few combination chemotherapy regimens including high-dose MTX for PCNSL that can be used in Japan. Other combination regimens that are reportedly effective for PCNSL (13-16) include anticancer drugs that are not approved by insurance in Japan (e.g. thiotepa other than use in ASCT, temozolomide, lomustine, and carmustine). However, few reports have described the outcomes of the R-MPV regimen in real-world settings.

When we searched the terms "R-MPV," "(MPV) AND (lymphoma)," and "(reduced dose whole brain) AND (lymphoma)" in PubMed on March 20, 2021, we found only two prospective trials (including the original trial) and two retrospective trials that reported the results of this regimen for PCNSL (Table 2) (1, 17-19). One of the trials only included patients $<60$ years old (17), and another omitted WBRT for 21 of $39(54 \%)$ patients, mainly because they were $\geq 71$ years old (18). To our knowledge, the present study is the first to evaluate the R-MPV plus ra-WBRT regimen for elderly patients in a real-world setting. 
Table 2.

\begin{tabular}{|c|c|c|c|c|c|c|}
\hline reference & study design & $\begin{array}{l}\text { Treated } \\
\text { No }\end{array}$ & $\begin{array}{l}\text { Median age } \\
\text { (range) }\end{array}$ & $\begin{array}{l}\text { median } \\
\text { follow up }\end{array}$ & PFS (ITT) & OS (ITT) \\
\hline 1 & prospective phase 2 & 52 & $60(30-79)$ & 67.2 & $3 y: 51 \%$ median $39.6 \mathrm{~m}$ & $3 y: 77 \%$ median $79.2 \mathrm{~m}$ \\
\hline 17 & retrospective & 10 & $54.5(47-59)^{\#}$ & 33.1 & $3 y: 56 \%$ median not reached & $3 y: 69 \%$ median not reached \\
\hline 18 & retrospective & $39 *$ & 73 & 42.4 & median not reached & median not reached \\
\hline 19 & prospective phase 2 & 22 & $51.5(31-67)$ & 11.3 & 1y: $94 \%$ median not reached & 1y: $68 \%$ median $19 \mathrm{~m}$ \\
\hline Our & retrospective & 10 & $69(62-77)$ & 44.4 & $3 y: 60 \%$ median $57.6 \mathrm{~m}$ & $3 y: 80 \%$ median $78.2 \mathrm{~m}$ \\
\hline
\end{tabular}

\#This study only enrolled patients under 60 years old.

*WBRT was omitted in 21 of 39 patients in this trial.

ITT: intent to treat, OS: overall survival, PFS: progression-free survival, R-MPV: rituximab methotrexate procarbazine vincristine, WBRT: whole-brain radiotherapy

The present study showed that the PFS and OS rates in our facility were good despite the patients' relatively old age and low PS. However, our actual results are not satisfactory, with only 1 patient (10\%) surviving without delayed neurotoxicity. This indicates the peculiar difficulty in evaluating treatment outcomes in patients with PSCNL, in whom adverse events of delayed neurotoxicity are a major problem. Large-scale real-world data on the delayed neurotoxicity-free PFS or quality of life-adjusted PFS will be required in the future.

The original prospective trial of R-MPV plus rd-WBRT of 23.4 Gy showed minimal delayed neurotoxicity among 12 patients who achieved a progression-free status and completed neuropsychological evaluations up to 48 months. However, only three elderly patients were included among them (1). Our study shows that delayed neurotoxicity remains a major problem for elderly patients with PCNSL (more than half of patients were affected, including a severely affected patient), even those receiving rd-WBRT at 23.4 Gy. Multiple reports have shown that patients' age is the main risk factor for an increased incidence of delayed neurotoxicity, which occurred in $19 \%$ to $83 \%$ of patients > 60 years old who received WBRT following MTX-based chemotherapy at an average of 3 to 52 months after completing treatment $(20,21)$. One report showed no marked difference in the quality-adjusted life expectancy between elderly patients with PCNSL undergoing chemotherapy alone and those undergoing chemotherapy plus WBRT (22). We should consider deferring WBRT until relapse for elderly patients, as has been attempted in clinical trials $(19,21)$. Even when WBRT is performed, we should consider keeping the dose at $<30 \mathrm{~Gy}$, even when proceeding to 45 Gy WBRT in the protocol. This is because WBRT at 45 Gy in elderly patients reportedly causes a high rate of neurological damage $(>70 \%)(24,25)$, and the outcomes in our patients were also severe.

We believe that it is important to maintain the RDI of high-dose MTX in order to avoid WBRT and a worsening treatment outcome. Notably, the initial dose of MTX was reduced in $40 \%$ of the patients in our study, and only $30 \%$ of the patients completed the regimen without MTX dose modification. Our study also shows that some clinicians re- duce the MTX dose by more than half due to concerns regarding the toxicity of high-dose MTX in elderly patients, but this decision is not based on clearly established criteria. The optimal MTX dose and schedule for elderly patients have yet to be determined. A large multicenter retrospective analysis recently suggested that maximizing the MTX-RDI and delivering consolidation in a subgroup of elderly patients may improve clinical outcomes (26). Tatarczuch et al. (27) reported good outcomes of R-MPV therapy (4-year PFS and 4-year OS of $82 \%$ and $77 \%$, respectively) in elderly patients ( $>60$ years old), with minimal dose reduction of MTX and only $11 \%$ receiving WBRT. High-dose MTXbased therapy is associated with an improved survival compared with regimens without high-dose MTX; MTX is thus the key drug in treating PCNSL. Several clinical trials have indicated that reasonable MTX doses in elderly patients are considered to range from 3 to $4 \mathrm{~g} / \mathrm{m}^{2}(16,23,28)$. Our study also showed that a high MTX-RDI $(>80 \%)$ tended to provide better outcomes and enabled a reduction in the intensity of WBRT. We should make efforts to maintain the RDI of high-dose MTX even in elderly patients.

The role of high-dose AraC in this regimen for elderly patients, however, is less clear. One randomized phase 2 trial showed that the addition of high-dose AraC to high-dose MTX provides improved outcomes with acceptable toxicity compared with high-dose MTX alone in patients $\leq 75$ years old (29). Another prospective phase 2 trial showed similar outcomes between elderly patients and young patients treated with high-dose AraC containing consolidation and omitting WBRT (13). However, one retrospective trial showed that extending high-dose AraC consolidation of the MPV-AAA regimen resulted in no notable improvement in the $\mathrm{CR}$ rate, $\mathrm{PFS}$, or OS but had a higher rate of hematotoxicity than the historical control of the MPV-A regimen among elderly patients (30). In the present study, the only patients who survived without relapse or neurotoxicity received the full dose of high-dose AraC without reduction. At present, we support the practice of administering high-dose AraC in elderly patients to the greatest extent possible.

Omitting WBRT may increase the risk of relapse. A randomized phase 3 trial indicated a benefit of WBRT in terms of the PFS but not the OS (2). We should therefore consider 
consolidation therapy of HCT-ASCT. Randomized phase 2 studies have demonstrated that ASCT and WBRT are both as effective as consolidation and that ASCT preserves or improves the cognitive function $(6,31)$. Omuro et al. (5) reported effective disease control and acceptable toxicity with R-MPV followed by ASCT (2-year PFS, 79\%; 2-year OS, $81 \%$ ). However, all of the above-mentioned ASCT studies showed an ASCT treatment-related mortality rate of approximately $3 \%$ to $12 \%$, even in patients $<60$ years old. Therefore, we hesitated to administer ASCT in the present study because of the relatively old age and low PS of our cohort. A recent pilot trial indicated the feasibility and effectiveness of high-dose MTX comprising induction followed by HCT (thiotepa plus 2-day busulfan). The median age of the patients was 74 (range, 69-79) years old, and all patients except 1 survived without relapse (32). In Japan, thiotepa was approved for HCT combined with busulfan for adult lymphoma in March 2020 following a clinical study of its safety (33). HCT-ASCT using such a regimen may thus be considered for select patients, even those $>60$ years old.

One limitation of this study is the small number of patients included from a single institution. Furthermore, our study was retrospective, most of the chemotherapy reductions and follow-up intervals were at the discretion of the attending physician, and response assessments were also left to each attending physician. In addition, delayed neurotoxicity was mostly evaluated based on patient complaints and MRI findings; objective cognitive tests were insufficiently performed. Therefore, it will be necessary to evaluate a larger number of patients with PCNSL from multiple centers in order to determine the optimal treatment strategy for realworld populations according to objective and uniform indicators.

In conclusion, the R-MPV regimen with ra-WBRT for elderly patients at our institution resulted in a good response rate and PFS and OS rates comparable with those in previous reports. However, the long-term outcome was not satisfactory because only one patient survived without relapse and delayed neurotoxicity. Delayed neurotoxicity, which leads to a decline in the quality of life, was observed even with rd-WBRT at $23.4 \mathrm{~Gy}$. We believe that it is important to endeavor to maintain MTX-RDI, postpone WBRT until relapse or minimize radiation dose, and choose consolidation of ASCT for elderly patients with PCNSL who are in a good condition. Individualized approaches based on objective indicators are needed in the future.

\section{Ethical approval}

All procedures performed in studies involving human participants were in accordance with the ethical standards of the institutional and/or national research committee and with the 1964 Declaration of Helsinki and its later amendments or comparable ethical standards. This study was approved by the Toyohashi Municipal Hospital review board.

The authors state that they have no Conflict of Interest (COI).

\section{Acknowledgement}

We would like to thank all staff involved in the patients' medical treatment. We also thank Michelle McRae, PhD and Angela Morben, DVM, ELS, from Edanz (https://jp.edanz.com/ac) for editing a draft of this manuscript.

\section{References}

1. Morris PG, Correa DD, Yahalom J, et al. Rituximab, methotrexate, procarbazine, and vincristine followed by consolidation reduceddose whole-brain radiotherapy and cytarabine in newly diagnosed primary CNS lymphoma: final results and long-term outcome. J Clin Oncol 31: 3971-3979, 2013.

2. Thiel E, Korfel A, Martus P, et al. High-dose methotrexate with or without whole brain radiotherapy for primary CNS lymphoma (GPCNSL-SG-1): a phase 3, randomised, non-inferiority trial. Lancet Oncol 11: 1036-1047, 2010.

3. Herrlinger U, Schäfer N, Fimmers R, et al. Early whole brain radiotherapy in primary CNS lymphoma: negative impact on quality of life in the randomized G-PCNSL-SG1 trial. J Cancer Res Clin Oncol 143: 1815-1821, 2017.

4. Omuro AMP, Ben-Porat LS, Panageas KS, et al. Delayed neurotoxicity in primary central nervous system lymphoma. Arch Neurol 62: 1595-1600, 2005.

5. Omuro A, Correa DD, DeAngelis LM, et al. R-MPV followed by high-dose chemotherapy with TBC and autologous stem-cell transplant for newly diagnosed primary CNS lymphoma. Blood 125: 1403-1410, 2015.

6. Ferreri AJM, Cwynarski K, Pulczynski E, et al. Whole-brain radiotherapy or autologous stem-cell transplantation as consolidation strategies after high-dose methotrexate-based chemoimmunotherapy in patients with primary CNS lymphoma: results of the second randomisation of the International Extranodal Lymphoma Study Group-32 phase 2 trial. Lancet Haematol 4: e510-e523, 2017.

7. Shah GD, Yahalom J, Correa DD, et al. Combined immunochemotherapy with reduced whole-brain radiotherapy for newly diagnosed primary CNS lymphoma. J Clin Oncol 25: 4730-4735, 2007.

8. Ichikara T, Kurozumi K, Michiue H, et al. Reduced neurotoxicity with combined treatment of high-dose methotrexate, cyclophosphamide, doxorubicin, vincristine and prednisolone (M-CHOP) and deferred radiotherapy for primary central nervous system lymphoma. Clin Neurol Neurosurg 127: 106-111, 2014.

9. Lachance DH, Brizel DM, Gockerman JP, et al. Cyclophosphamide, doxorubicin, vincristine, and prednisone for primary central nervous system lymphoma: short-duration response and multifocal intracerebral recurrence preceding radiotherapy. Neurology 44: 1721-1727, 1994.

10. Abrey LE, Batchelor TT, Ferreri AJ, et al. Report of an international workshop to standardize baseline evaluation and response criteria for primary CNS lymphoma. J Clin Oncol 23: 5034-5043, 2005.

11. Eisenhauer EA, Therasse P, Bogaerts J, et al. New response evaluation criteria in solid tumours: revised RECIST guideline (version 1.1). Eur J Cancer 45: 228-247, 2009.

12. Kanda Y. Investigation of the freely available easy-to-use software "EZR" for medical statistics. Bone Marrow Transplant 48: 452458, 2013.

13. Rubenstein JL, Hsi ED, Johnson JL, et al. Intensive chemotherapy and immunotherapy in patients with newly diagnosed primary CNS lymphoma: CALGB 50202 (Alliance 50202). J Clin Oncol 31: 3061-3068, 2013.

14. Ferreri AJ, Cwynarski K, Pulczynski E, et al. Chemoimmunotherapy with methotrexate, cytarabine, thiotepa, and rituximab (MATRix regimen) in patients with primary CNS lymphoma: results of 
the first randomisation of the International Extranodal Lymphoma Study Group-32 (IELSG32) phase 2 trial. Lancet Haematol 3: e 217-e227, 2016.

15. Bromberg J, Issa $S$, Bakunina $K$, et al. Rituximab in patients with primary CNS lymphoma (HOVON 105/ALLG NHL 24): a randomised, open-label, phase 3 intergroup study. Lancet Oncol 20: 216-228, 2019.

16. Illerhaus G, Marks R, Muller F, et al. High-dose methotrexate combined with procarbazine and CCNU for primary CNS lymphoma in the elderly: results of a prospective pilot and phase II study. Ann Oncol 20: 319-325, 2009.

17. Hattori K, Sakata-Yanagimoto M, Okoshi Y, et al. A single institutional retrospective evaluation for younger patients with primary central nervous lymphomas on a modified R-MPV regimen followed by radiotherapy and high dose cytarabine. J Clin Exp Hematop 57: 41-46, 2017.

18. Sasaki N, Kobayashi K, Saito K, et al. Consecutive singleinstitution case series of primary central nervous system lymphoma treated by R-MPV or high-dose methotrexate monotherapy. Jpn J Clin Oncol 50: 999-1008, 2020.

19. Adhikari N, Biswas A, Gogia A, et al. A prospective phase II trial of response adapted whole brain radiotherapy after high dose methotrexate based chemotherapy in patients with newly diagnosed primary central nervous system lymphoma-analysis of acute toxicity profile and early clinical outcom. J Neurooncol 139: 153166, 2018.

20. Daras M, DeAngelis LM. Management of elderly patients with primary central nervous system lymphoma. Curr Neurol Neurosci Rep 13: 344, 2013.

21. Siegal T, Bairey O. Primary CNS lymphoma in the elderly: the challenge. Acta Haematol 141: 138-145, 2019.

22. Prica A, Chan K, Cheung MC. Combined modality therapy versus chemotherapy alone as an induction regimen for primary central nervous system lymphoma: a decision analysis. Br J Haematol 158: 600-607, 2012.

23. Omuro A, Chinot O, Taillandier L, et al. Methotrexate and temozolomide versus methotrexate, procarbazine, vincristine, and cytarabine for primary CNS lymphoma in an elderly population: an intergroup ANOCEF-GOELAMS randomised phase 2 trial. Lancet Haematol 2: e251-e259, 2015.

24. Gavrilovic IT, Hormigo A, Yahalom J, DeAngelis LM, Abrey LE. Long-term follow-up of high-dose methotrexate-based therapy with and without whole brain irradiation for newly diagnosed pri- mary CNS lymphoma. J Clin Oncol 24: 4570-4574, 2006.

25. Abrey LE, Yahalom J, DeAngelis LM. Treatment for primary CNS lymphoma: the next step. J Clin Oncol 18: 3144-3150, 2000.

26. Martinez-Calle N, Poynton E, Alchawaf A, et al. Outcomes of older patients with primary central nervous system lymphoma treated in routine clinical practice in the UK: methotrexate dose intensity correlates with response and survival. Br J Haematol 190: 394-404, 2020.

27. Tatarczuch M, Paul E, Gilberston M, et al. Excellent outcomes in older patients with primary CNS lymphoma treated with R-MPV/ cytarabine without whole brain radiotherapy or autologous stem cell transplantation therapy. Leuk Lymphoma 62: 112-117, 2020.

28. Deckert M, Engert A, Bruck W, et al. Modern concepts in the biology, diagnosis, differential diagnosis and treatment of primary central nervous system lymphoma. Leukemia 25: 1797-1807, 2011.

29. Ferreri AJ, Reni M, Foppoli M, et al. High-dose cytarabine plus high-dose methotrexate versus high-dose methotrexate alone in patients with primary CNS lymphoma: a randomised phase 2 trial. Lancet 374: 1512-1520, 2009.

30. Houillier C, Ghesquières $\mathrm{H}$, Chabrot $\mathrm{C}$, et al. Rituximab, methotrexate, procarbazine, vincristine and intensified cytarabine consolidation for primary central nervous system lymphoma (PCNSL) in the elderly: a LOC network study. J Neurooncol 133: 315-320, 2017.

31. Houillier C, Taillandier L, Dureau S, et al. Radiotherapy or autologous stem-cell transplantation for primary CNS lymphoma in patients 60 years of age and younger: results of the intergroup ANOCEF-GOELAMS randomized phase II PRECIS study. J Clin Oncol 37: 823-833, 2019.

32. Schorb E, Kasenda B, Ihorst G, et al. High-dose chemotherapy and autologous stem cell transplant in elderly patients with primary CNS lymphoma: a pilot study. Blood Adv 4: 3378-3381, 2020.

33. Kondo E, Ikeda T, Goto H, et al. Pharmacokinetics of thiotepa in high-dose regimens for autologous hematopoietic stem cell transplant in Japanese patients with pediatric tumors or adult lymphoma. Cancer Chemother Pharmacol 84: 849-860, 2019.

The Internal Medicine is an Open Access journal distributed under the Creative Commons Attribution-NonCommercial-NoDerivatives 4.0 International License. To view the details of this license, please visit (https://creativecommons.org/licenses/ by-nc-nd/4.0/).

\section{(C) The Japanese Society of Internal Medicine Intern Med Advance Publication}

